\title{
Black-box modeling of nonlinear system using evolutionary neural NARX model
}

\author{
Nguyen Ngoc Son, Nguyen Duy Khanh, Tran Minh Chinh \\ Faculty of Electronics Technology, Industrial University of Ho Chi Minh City, Viet Nam
}

\begin{tabular}{|c|c|}
\hline Article Info & ABSTRACT \\
\hline Article history: & \multirow{6}{*}{$\begin{array}{l}\text { Nonlinear systems with uncertainty and disturbance are very difficult to } \\
\text { model using mathematic approach. Therefore, a black-box modeling } \\
\text { approach without any prior knowledge is necessary. There are some } \\
\text { modeling approaches have been used to develop a black box model such as } \\
\text { fuzzy logic, neural network, and evolution algorithms. In this paper, an } \\
\text { evolutionary neural network by combining a neural network and a modified } \\
\text { differential evolution algorithm is applied to model a nonlinear system. The } \\
\text { feasibility and effectiveness of the proposed modeling are tested on a } \\
\text { piezoelectric actuator SISO system and an experimental quadruple tank } \\
\text { MIMO system. }\end{array}$} \\
\hline Received May 12, 2018 & \\
\hline Revised Dec 18, 2018 & \\
\hline Accepted Dec 29, 2018 & \\
\hline Keywords: & \\
\hline Differential evolution & \\
\hline
\end{tabular}

Copyright $@ 2019$ Institute of Advanced Engineering and Science. All rights reserved.

\section{Corresponding Author:}

Nguyen Ngoc Son,

Faculty of Electronics Technology,

Industrial University of Ho Chi Minh City,

12 Nguyen Van Bao Street, 4 Ward, Go Vap District, Ho Chi Minh City, Viet Nam.

Email: nguyenngocson@iuh.edu.vn

\section{INTRODUCTION}

Mathematical modeling of systems is a very common methodology in engineering. It is used both as a means for achieving deeper knowledge about a system and as a basis for simulations or for the design of controllers. However, in practice, it is not easy to obtain an accurate mathematical model of a nonlinear system because of a lack of knowledge of the system and disturbance impact to system maybe still unknown. In these cases, system identification can be a way of solving the modeling problem. System identification deals with the problem of how to estimate a model of a system from measured input and output signals. Black-Box nonlinear modeling approaches include non-parametric methods, such as neural networks, fuzzy logic, genetic algorithm etc., [1] which do not require a priori knowledge.

Recently, the multilayer feed-forward neural network (MLP) has been widely used in nonlinear system identification. As we know, the performance of a neural network is dependent on the training process. A popular training algorithm is the back-propagation. However, the back-propagation (BP) algorithm only perform a local search around the initial values and provide local optimizations [2], [3]. Therefore the metaheuristic algorithms based training procedures are considered as promising alternatives. The meta-heuristic algorithms generate global optimum because of their ability to search the global solution space and avoiding falling into a locally optimal solution. For example, a genetic algorithm [4]-[6] is used for turning the structure and parameters of neural networks. Papers [7]-[9] introduced the particle swarm optimization (PSO) to train the neural network model. Valian et al. [10] proposed the improved cuckoo search algorithm for training feed-forward neural network for two benchmark classification problems. Although these proposed methods obtained good results, two challenges that need to be further improved in training neural network are how to find the global optimal solution and how to achieve a fast convergence speed. 
Among meta-heuristic algorithms, the differential evolution (DE) technique was regarded as a promising stochastic global optimization algorithm. The DE algorithm was first studied and published by Storn R. and Price K.V. [11]. Its advantages are as follows: the simple and straightforward features in installation, better performance, fewer parameters involved, and low complexity. Over recent years, the DE technique had been applied to learn a neural model via optimizing real and constrained integer weighting values [12]-[15]. Nonetheless, DE technique gets the slow convergence speed for high-sized optimization tasks. Furthermore, DE technique often seems easy to fall into a stagnant situation that is a state that the optimum searching procedure stagnates before obtaining a global optimum solution. Some suggestions on how to improve the DE performance on the training neural network have been studied [16]-[20]. Paper [21] proposed the hybrid modified differential evolution plus back-propagation algorithm (MDE-BP) for training neural network model. The performance and efficiency of the proposed MDE-BP training method are tested on identifying some benchmark nonlinear systems. However, paper [21] only perform for SISO nonlinear identification system. Therefore, this paper continuously studies the proposed MDE-BP training algorithm in [21] to train a neural network for a general nonlinear identification and its approach to model a piezoelectric actuator SISO system and an experimental quadruple tank MIMO system. These systems are suitable to test the effectiveness of the proposed method because of its hysteresis behavior and highly nonlinearity characteristic.

The rest of this paper is organized as follows. Section 2 introduces the proposed MDE-BP training neural NARX model for MIMO nonlinear system identification. Section 3 presents the results and discussion. Where Section 3.1 introduces to model a piezoelectric actuator SISO system. Section 3.2 introduces an experimental quadruple tank setup and the identification results. Finally, Section 4 contains some conclusions.

\section{RESEARCH METHOD}

Evolutionary neural NARX (ENN) model is proposed in [21] by connecting the Multi-Layer Perceptron Neural Networks (MLP) model and NARX structure. The weight value of ENN model is optimized by a hybridizing modified differential evolution and backpropagation training (MDE-BP) algorithms. Based on this combination, the ENN model obtains a strongly approximating feature of the MLP scheme and a powerful predictive feature of the NARX structure. The ENN model structure, which is used for nonlinear MIMO system identification, is illustrated in Figure 1.

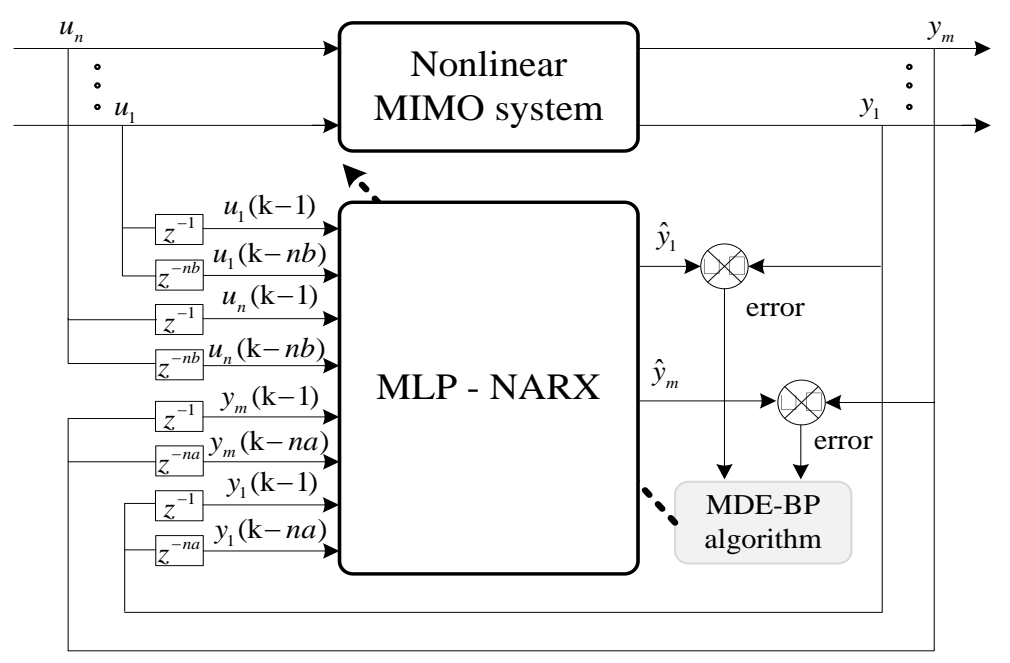

Figure 1. The ENN model for a nonlinear system identification

Where, $u(k)$ and $y(k)$ represent the input and output signals of nonlinear MIMO system; $n a$ and $n b$ are order of output $y(k)$ and input $u(k)$, respectively; $q$ is the backward time shift operator defined as $q^{-1} u(\mathrm{k})$ $=u(k-1) . v_{j n}$ is the weight of input layer, $v_{j 0}$ is the weight of bias input layer, $\mathrm{w}_{1 \mathrm{j}}$ is the weight of hidden layer, $\mathrm{w}_{10}$ is the weight of bias hidden layer; $f_{j}(:)$ is sigmoid function at hidden layer, $F(:)$ is linear activation function at output layer; the dimension number $D$ is the sum of the number of weights and thresholds of the 
neural NARX model; $u(\mathrm{k})=\left[u_{1}(k), \mathrm{u}_{2}(k), \ldots, \mathrm{u}_{n}(k)\right]$ is the input signal vector and $y(\mathrm{k})=\left[y_{1}(k), \mathrm{y}_{2}(k), \ldots, \mathrm{y}_{m}(k)\right]$ is the output signal vector of nonlinear MIMO system. We determine the prediction output as follows:

$$
\hat{y}(k, \theta)=F\left(\sum_{i=1}^{j} w_{1 i} f_{i}\left(\sum_{l=1}^{n} u_{i l} \varphi_{l}(k)+v_{l 0}\right)+w_{10}\right)
$$

Where, $j(k)$ is the regression vector and $q$ is the weight vector, defined by

$$
\begin{aligned}
& \varphi(k)=\left[\varphi_{1}(k), \ldots, \varphi_{n}(k)\right]^{T}=\left[\begin{array}{l}
y(k-1), \ldots, y(k-n a), y_{m}(k-1), \ldots, y_{m}(k-n a), \\
u(k-1), \ldots, u(k-n b), u_{n}(k-1), \ldots, u_{n}(k-n b)
\end{array}\right]^{T} \\
& \theta=\left[v_{10}, \ldots, v_{10}, v_{j 0}, \ldots, v_{j n}, w_{10}, \ldots, w_{1 j}\right]^{T}=\left[w_{1}, \ldots, w_{D}\right]^{T}
\end{aligned}
$$

The modified differential evolution training algorithm is used to optimally generate the value of the weight of the neural NARX model. The output of the neural NARX model is a prediction function $\hat{y}(k \mid q)$ of the synaptic weight vector $\theta$ and regression vector $\varphi(k)$.

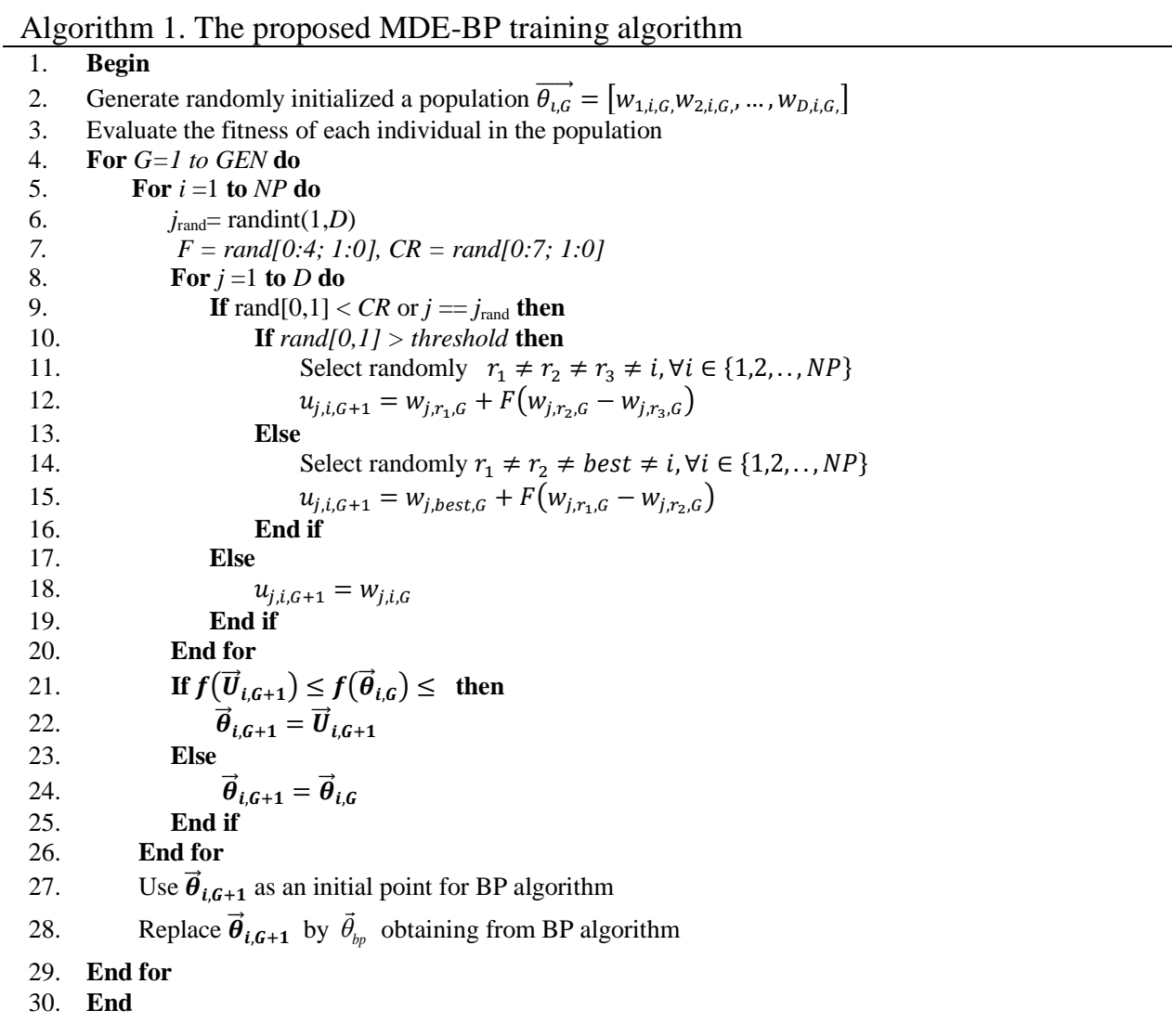

In the training process, both the input signal $u(k)$ and the output signal $y(k)$ of nonlinear MIMO system are known and the synaptic weight vector $\theta$ is adapted to obtain appropriate functional mappings from the input $u(k)$ to the output $y(k)$. Generally, the adaptation process can be carried out by minimizing the network error function $E_{N}$ which is based on the introduction of a measure of closeness in terms of a mean square error (MSE) criterion.

$$
E_{N}\left(\theta, Z^{N}\right)=\frac{1}{2 N} \sum_{k=1}^{N}[y(k)-\hat{y}(k \mid \theta)]^{T}[y(k)-\hat{y}(k \mid \theta)]
$$


Where the training data $Z^{N}$ is specified by $Z^{N}=\{[y(k), u(k)] \mid k=1,2, \ldots, N\}$. The optimization goal is to minimize the objective function $\mathrm{E}_{\mathrm{N}}$ by optimizing the values of the network weights $q=\left[w_{1}, w_{2}, \ldots ., w_{D}\right]$ by using the MDE-BP algorithm which is described in details at paper [21]. The working steps involved in employing MDE-BP training algorithm as Algorithm 1.

\section{RESULTS AND ANALYSIS}

All of the modelings is performed by Matlab version $2013 \mathrm{~b}$ on an Intel Core i3 computer with a clock rate of $2.53 \mathrm{GHz}$ and $2.00 \mathrm{~GB}$ of RAM.

\subsection{Modeling the hysteresis behavior of the piezoelectric actuator}

Micropositioning stages using piezoelectric actuator are widely used in a variety of applications such as microgripper robot [22], energy harvesting [23], MEMs [24] and so on. The piezoelectric actuator possesses a higher displacement accuracy, larger generation force and higher response speed than other types of actuators. However, the nonlinearities hysteresis of the piezoelectric actuator can greatly degrade the positioning accuracy of micropositioning systems [25], [26]. Therefore, modeling precisely and identifying parameter effectively for piezoelectric actuator still remain a challenging problem nowadays. To describe the hysteresis behavior of the piezoelectric actuator, many mathematical models have been proposed such as Prandtl-Ishlinskii, Preisach model, Bouc-Wen model, KP model, Duhem model and so on [27]. However, the difficulties in determining the parameters of the hysteresis model limit its application in practice.
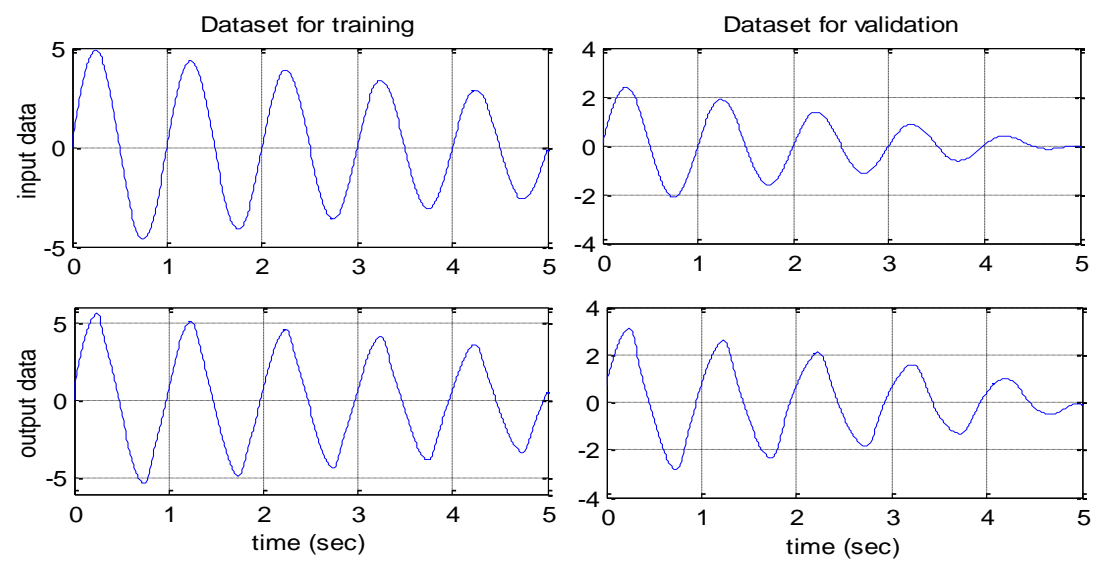

Figure 2. Selection of training data for estimating and validating purposes

To overcome this problem, paper [25] proposed a parameter identification method based on artificial neural networks for the Duhem hysteresis model. The mathematical model of the piezoelectric actuator based Duhem model in [25] can be described as

$$
\left\{\begin{array}{l}
m \ddot{y}(t)+n \dot{y}(t)+k y(t)=k(d u(t)-w(t)) \\
\dot{w}(t)=\alpha|\dot{u}(t)|(c u(t)-w(t))+b \dot{u}(t)
\end{array}\right.
$$

where $m$ is the equivalent mass; $n$ is the equivalent damping; $k$ is the equivalent stiffness; $\mathrm{u}(\mathrm{t})$ is the input voltage; $y(t)$ is the output displacement; $w(t)$ is the hysteretic state variable; parameters $\alpha, b, c$ controls the shapes and amplitudes of the hysteresis loop; and $d$ is the effective piezoelectric coefficient of the actuator. In this paper, the ENN model trained MDE-BP algorithm is proposed to identify the nonlinearities Duhem hysteresis of the piezoelectric actuator.

Firstly, the above system (5) is implemented in MATLAB. The training data set and validating data set are collected form system (5) and shown in Figure 2. Secondly, the ENN structure is selected by linking the 3-layer MLP with S1 hidden nodes and the $2^{\text {nd }}$ order NARX structure, is presented with the regression and prediction functions, respectively. Selecting parameter $\mathrm{S} 1=5, \mathrm{NP}=62$ and $\mathrm{GEN}=10000$. 


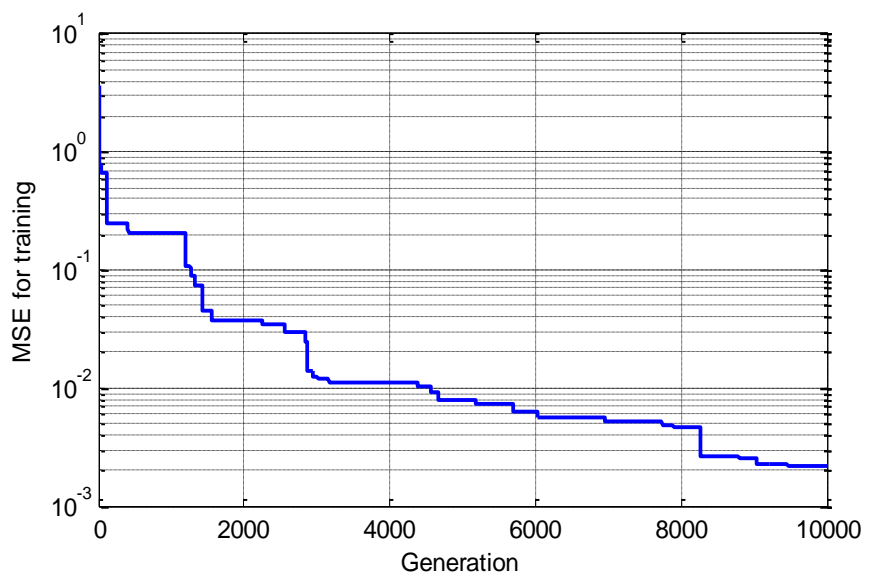

Figure 3. Convergence rate in training
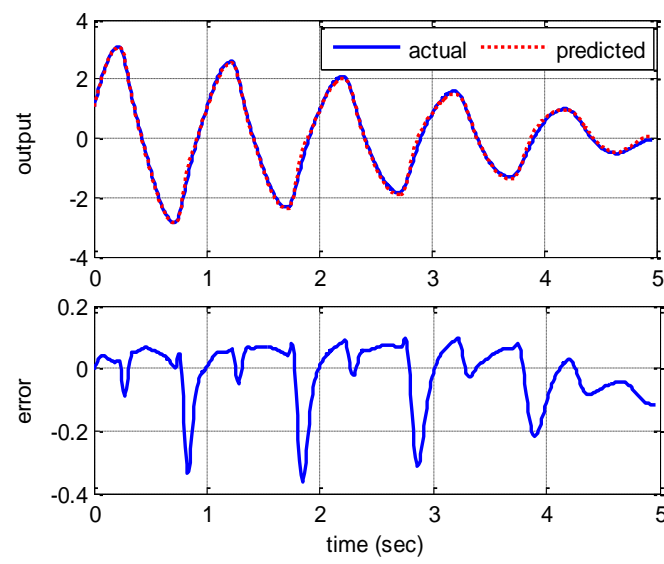

Figure 4. Identification performance

Finally, the performance identification using the proposed ENN model is conducted. Figure 3 shows the convergence rates of MDE-BP-ENN in the training process. Figure 4 demonstrates the errors between the predicted output of the neural network and the formula output of Duhem model. The results indicate the proposed method can be used to identify and predict the hysteresis behavior of the piezoelectric actuator.

\subsection{Modeling a quadruple tank system}

In practice, a common tank system consists of tanks, pumps, piping, valves, liquid level sensors. During operation, depending on the technology requirements and load requirements, the valves can change the aperture, or the liquid type in the tanks can change. This leads to a nonlinear characteristic of the system that can vary. Moreover, the parameters of the liquid tank system such as the size and discharge coefficient of the valves, the power factor of the pump in some cases may be still unknown. Therefore, it is not easy to obtain an accurate theoretical model of a quadrature tank system.

To overcome the disadvantages of the theoretical modeling method, some identification approaches have been investigated. Paper [28] proposed an adaptive fuzzy model predictive control based on the ant colony optimization (ACO) for nonlinear process system. Paper [29] used a radial basis function neural network as the prediction function due to its approximation ability. Then, a nonlinear model predictive control method was proposed to guarantee the system stability and compensate the network-induced delays and packet dropouts. Iplikci [30] proposed a support vector machine (SVM) approach to the generalized predictive control (GPC) of an experimental three-tank system. One advantage of the black-box modeling approach is that it is unnecessary to precisely analyze its internal mechanism or the relationship among physical variables. In this paper, the ENN model trained MDE-BP algorithm is proposed to identify the black-box modeling of a quadruple tank system.

\subsubsection{Physical model}

The quadruple tank system which is described in Figure 5 is a nonlinear plant with strong coupling and large time delay. The quadruple tank plant can be seen as a system with two inputs, the voltages of the pumps, and two outputs, the water levels of the lower tanks.

Where $L_{i}$ is the liquid level in tank i; $D_{t i}$ is the outlet cross-sectional area of tank i; $D_{o i}$ is the crosssectional area of tank $\mathrm{i} ; \gamma_{i}$ is the portion of the flow into the upper tank from pump $\mathrm{i} ; u_{j}$ is the speed setting of pump $\mathrm{j}$, with the corresponding gain $\mathrm{k}_{\mathrm{j}}$. A mathematical model based on physical data together with its linearization around an operating point is derived in Johansson [31]. 


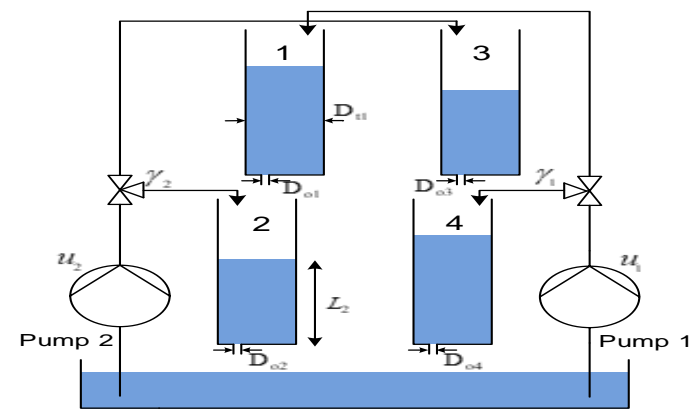

Figure 5. Schematic of the quadruple-tank system

\subsubsection{Experimental quadruple tank setup}

In this part, the experimental quadruple tank system has been studied. Figure 6 describes the diagram block of the experimental quadruple tank system. Table 1 describes in detail the physical parameters of the tank model system.

Table 1. The Parameters of the Quadruple-tank System

\begin{tabular}{lcc}
\hline \multicolumn{4}{c}{ Parameters } & Value & Unit \\
\hline The outlet cross-sectional area of the tank $\left(D_{t 1}, D_{t 2}, D_{t 3}, D_{t 4}\right)$ & 4.6 & $\mathrm{~cm}$ \\
The cross-sectional area of tank 1 and $3\left(D_{o 1}, D_{\mathrm{o} 3}\right)$ & 0.8 & $\mathrm{~cm}$ \\
The cross-sectional area of tank 2 and $4\left(D_{o 2}, D_{\mathrm{o} 4}\right)$ & 0.65 & $\mathrm{~cm}$ \\
The portion of the flow into the upper tank from pump 1, $\gamma_{1}$ & 0.7 & ------ \\
The portion of the flow into the upper tank from pump 2, $\gamma_{2}$ & 0.65 & ------ \\
The corresponding gain of Pump, $k_{p}$ & 6.9 & $\mathrm{~cm}^{3} / \mathrm{s} / \mathrm{V}$ \\
The speed setting of the pump, $\left(u_{1}, u_{2}\right)$ & ----- & $\mathrm{V}$ \\
\hline
\end{tabular}

The pump used in the quadruple-tank system is an Ogihara engine with a $24 \mathrm{~V}$ supply and a pump flow rate of 10 liters per minute. In fact, the motors pump water into the tank in the operating range of $30 \%$ $70 \%$ (Vmax). The Freescale Semiconductor's Strain Gauge MPX10 is used to measure water levels in tanks. Details of the equipment parameters of the quadruple-tank system are described in Table 2.

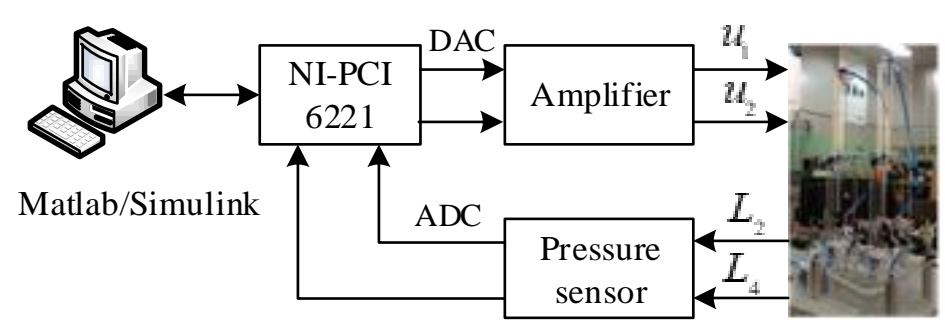

Figure 6. Diagram of the experimental quadruple tank system

Table 2. The parameters of Pump and MPX10

\begin{tabular}{cll}
\hline Equipment & & \multicolumn{1}{c}{ Parameters } \\
\hline \multirow{2}{*}{ Pump } & - & Supply voltage: $24 \mathrm{VDC}$ \\
& - & Flow rate: 10 liters/minute \\
& - & Power consumption: $26 \mathrm{~W}$ \\
MPX10 sensor & - & Type of MPX10DP CASE $344 \mathrm{C}$. \\
& - & Supply voltage: 3-6 VDC \\
& - & Range: $0-10 \mathrm{kPa}$ \\
\hline
\end{tabular}




\subsubsection{Identification inverse model results}

In fact, the inverse predictive model plays an important role in the design of model-based control. In this article, the inverse model of the quadruple-tank system is identified by using the ENN model. The procedure consists of four basic steps as follows:

Firstly, the experimental input-output data set, applied for training and validating purposes of the ENN based MDE-BP training algorithm, is collected from the quadruple-tank system. The input data set consists of the input signal as the voltage for the pump motor and the output signal of tank water level 2 and tank 4 collected from the experimental model described in Figure 7. Which, $u_{1}(k)$ and $u_{2}(k)$ are the voltage signal for the pump 1 and pump 2. $L_{2}(k)$ and $L_{4}(k)$ is the water level in tank 2 and tank 4 . The dataset with the input voltage ratio has a change in amplitude from 0 to 1 and the change frequency described in Figure 7 will be used to estimate and evaluate the MDE-ENN prediction model. Where, Figure 7(a) is used to estimate the model, Figure 7(b) is used to evaluate the model.

(a)
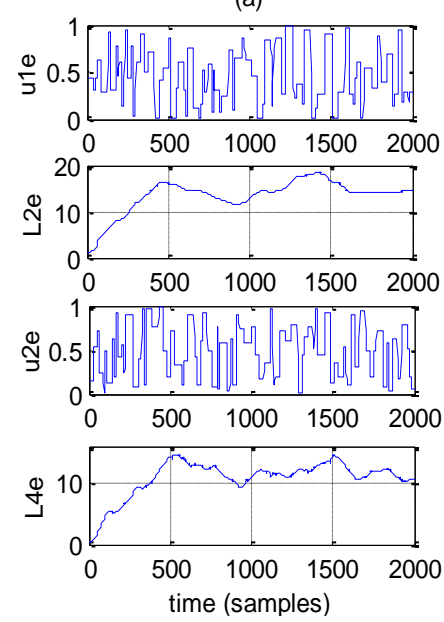

(b)
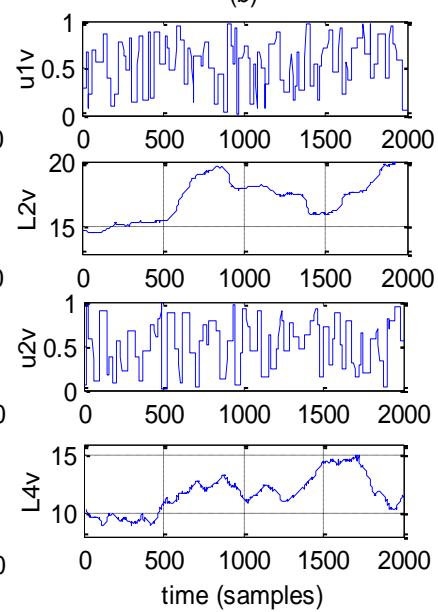

Figure 7. Experimental dataset for training and validation of the ENN model

Secondly, the ENN structure is selected by linking the 3 -layered MLP model with the $1^{\text {st }}$ order NARX structure and described in Figure 8. In this case, the number of hidden neurons is $\mathrm{S} 1=12$, population size $\mathrm{NP}=120$, number of generations of trained GEN $=700$, the coefficient of learning BP $\eta=0.000001$ and number of iterations for BP iter $_{3}=5$.

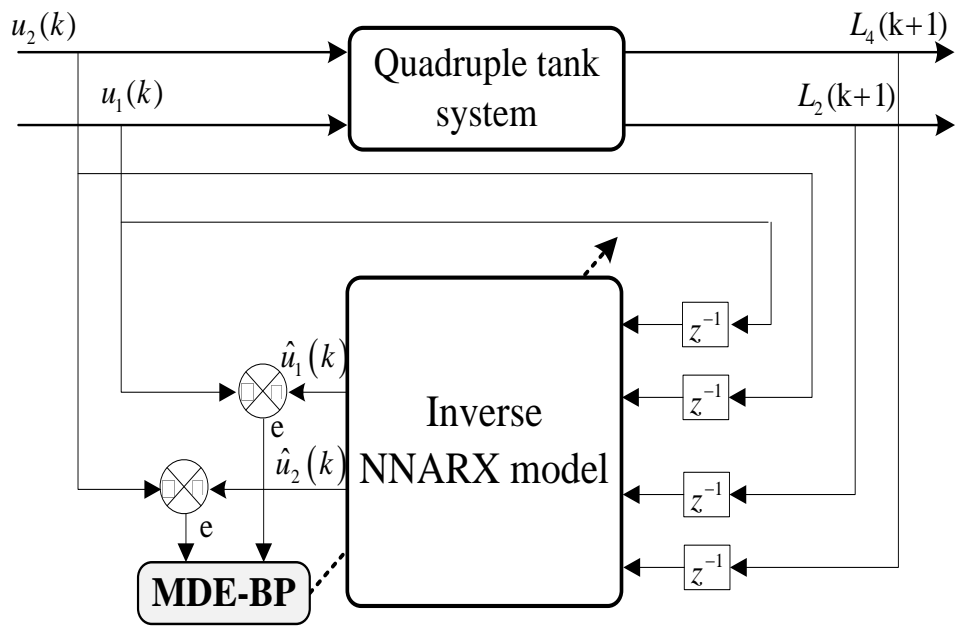

Figure 8. The inverse model of the quadruple-tank system 
Finally, the estimation and validation process are conducted to identify the ENN model. Figure 9 shows the performance of MDE-BP-ENN based on the values of MSE in the training process. Figure 10 shows the performance of MDE-BP-ENN based on the average values of MSE on the validation process. Based on the above results, we see that the ENN prediction model with weightings optimized by the MDEBP algorithm has been identified and predicted fairly accurately the inverse dynamic properties of the quadruple-tank system.
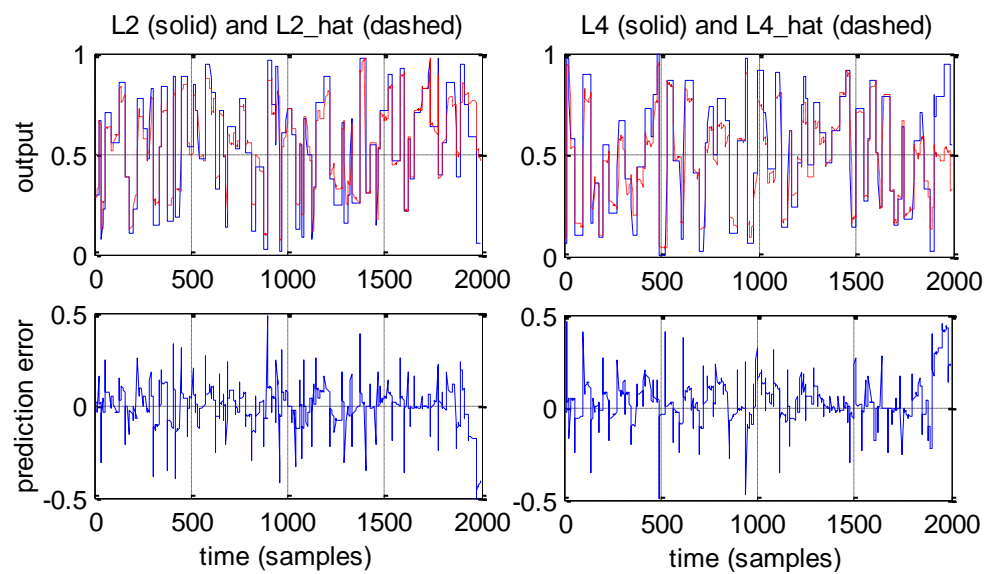

Figure 9. The MSE value on the training process
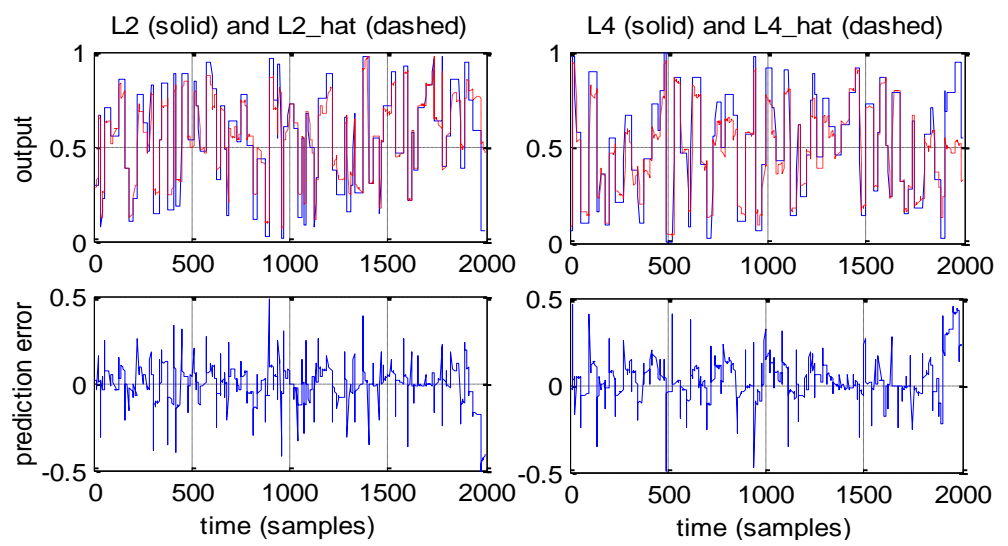

Figure 10. The MSE value on the validation process

\section{CONCLUSION}

This paper introduces how to modeling a nonlinear SISO and MIMO system based on input-output data using the proposed Evolutionary Neural NARX (ENN) model. The ENN scheme is used by connecting the Multi-Layer Perceptron Neural Networks (MLP) model and NARX structure. The weight value of ENN model is optimized by hybridizing a modified differential evolution and backpropagation training (MDE-BP) algorithms. The effectiveness of ENN model is tested on modeling a piezoelectric actuator and a quadruple tank system. The results prove that the ENN model has the ability to learn the dynamics model to reduce the tracking error to nearly zero. The results will be used to initialize the design of the model-based controller for nonlinear systems in the future research.

\section{ACKNOWLEDGEMENTS}

The study was supported by Science and Technology Incubator Youth Program, managed by the Center for Science and Technology Development, Ho Chi Minh Communist Youth Union, the contract number is "13/2018/HĐ-KHCN-VU". 


\section{REFERENCES}

[1] Ljung, Lennart, "System identification," Signal analysis and prediction, pp. 163-173, 1998.

[2] Wang, Lin, Yi Zeng, and Tao Chen, "Back propagation neural network with adaptive differential evolution algorithm for time series forecasting." Expert Systems with Applications, vol 42(2), pp. 855-863, 2015.

[3] Piotrowski, Adam P., "Differential evolution algorithms applied to neural network training suffer from stagnation." Applied Soft Computing, vol. 21, pp. 382-406, 2014.

[4] Yu, Feng, and Xiaozhong Xu, "A short-term load forecasting model of natural gas based on optimized genetic algorithm and improved BP neural network," Applied Energy, vol. 134, pp. 102-113, 2014.

[5] Leung, Frank Hung-Fat, et al., "Tuning of the structure and parameters of a neural network using an improved genetic algorithm," IEEE Transactions on Neural networks, vol. 14(1), pp. 79-88, 2003.

[6] Asadi, Ehsan, et al. "Multi-objective optimization for building retrofit: A model using genetic algorithm and artificial neural network and an application," Energy and Buildings, vol. 81, pp. 444-456, 2014.

[7] Song, Ying, Zengqiang Chen, and Zhuzhi Yuan, "New chaotic PSO-based neural network predictive control for nonlinear process," IEEE transactions on neural networks, vol. 18(2), pp. 595-601, 2007.

[8] Bashir Z. A., and M. E. El-Hawary, "Applying wavelets to short-term load forecasting using PSO-based neural networks," IEEE transactions on power systems, vol. 24(1), pp. 20-27, 2009.

[9] Gordan, Behrouz, et al, "Prediction of seismic slope stability through combination of particle swarm optimization and neural network," Engineering with Computers, 32(1), pp. 85-97, 2016.

[10] Valian, Ehsan, Shahram Mohanna, and Saeed Tavakoli, "Improved cuckoo search algorithm for feedforward neural network training," International Journal of Artificial Intelligence \& Applications, vol. 2(3), pp. 36-43, 2011.

[11] Storn, Rainer, and Kenneth Price, "Differential evolution-a simple and efficient heuristic for global optimization over continuous spaces," Journal of global optimization, vol. 11(4), pp. 341-359, 1997.

[12] Ilonen, Jarmo, Joni-Kristian Kamarainen, and Jouni Lampinen, "Differential evolution training algorithm for feedforward neural networks," Neural Processing Letters, vol. 17(1), pp. 93-105, 2003.

[13] Baştürk, Alper, and Enis Günay, "Efficient edge detection in digital images using a cellular neural network optimized by differential evolution algorithm," Expert Systems with Applications, vol. 36(2), pp. 2645-2650, 2009.

[14] Subudhi, Bidyadhar, and Debashisha Jena, "A differential evolution based neural network approach to nonlinear system identification," Applied Soft Computing, vol. 11(1), pp. 861-871, 2011.

[15] Chauhan, Nikunj, Vadlamani Ravi, and D. Karthik Chandra, "Differential evolution trained wavelet neural networks: Application to bankruptcy prediction in banks," Expert Systems with Applications, vol. 36(4), pp.7659-7665, 2009.

[16] Wang, Lin, Yi Zeng, and Tao Chen, "Back propagation neural network with adaptive differential evolution algorithm for time series forecasting," Expert Systems with Applications vol. 42(2), pp. 855-863, 2015.

[17] Liu, Wei, et al., "Neural network based on self-adaptive differential evolution for ultra-short-term power load forecasting," International conference on intelligent computing. Springer, Cham, 2014.

[18] Chen, Cheng-Hung, Cheng-Jian Lin, and Chin-Teng Lin, "Nonlinear system control using adaptive neural fuzzy networks based on a modified differential evolution." IEEE Transactions on Systems, Man, and Cybernetics, Part C (Applications and Reviews), vol. 39(4), pp. 459-473, 2009,

[19] Slowik, Adam, "Application of an adaptive differential evolution algorithm with multiple trial vectors to artificial neural network training," IEEE Transactions on Industrial Electronics, 58(8), pp. 3160-3167, 2011.

[20] Kumar, Jitendra, and Ashutosh Kumar Singh, "Workload prediction in cloud using artificial neural network and adaptive differential evolution," Future Generation Computer Systems, vol. 81, pp. 41-52, 2018.

[21] Nguyen, Son Ngoc, Vinh Ho- Huu, and Anh Pham Huy Ho, "A neural differential evolution identification approach to nonlinear systems and modelling of shape memory alloy actuator," Asian Journal of Control, 2018.

[22] Nah, S. K., and Z. W. Zhong, "A microgripper using piezoelectric actuation for micro-object manipulation," Sensors and Actuators A: Physical, vol. 133(1), pp. 218-224, 2007.

[23] Bowen, C. R., et al., "Piezoelectric and ferroelectric materials and structures for energy harvesting applications," Energy \& Environmental Science, vol. 7(1), pp. 25-44, 2014.

[24] Nguyen, Cuong H., Ulrik Hanke, and Einar Halvorsen, "Actuation of Piezoelectric Layered Beams with d31 and d33 Coupling," IEEE Transactions on Ultrasonics, Ferroelectrics, and Frequency Control, 2018.

[25] Wang, Geng, and Guoqiang Chen, "Identification of piezoelectric hysteresis by a novel Duhem model based neural network," Sensors and Actuators A: Physical, vol. 264, pp. 282-288, 2017.

[26] Mao, Xuefei, et al., "A Hybrid Feedforward-Feedback Hysteresis Compensator in Piezoelectric Actuators Based on Least-Squares Support Vector Machine," IEEE Transactions on Industrial Electronics, vol.65(7), pp. 5704-5711, 2018.

[27] Krasnosel'skii, Mark A., and Aleksei V. Pokrovskii, "Systems with hysteresis," Springer Science \& Business Media, 2012

[28] Bououden S., Mohammed Chadli, and Hamid Reza Karimi, "An ant colony optimization-based fuzzy predictive control approach for nonlinear processes," Information Sciences, vol. 299, pp. 143-158, 2015.

[29] Wang, Tong, Huijun Gao, and Jianbin Qiu, "A combined adaptive neural network and nonlinear model predictive control for multirate networked industrial process control," IEEE Transactions on Neural Networks and Learning Systems, vol. 27(2), pp. 416-425, 2016.

[30] Iplikci, Serdar, "A support vector machine-based control application to the experimental three-tank system," ISA transactions, vol. 49(3), pp. 376-386, 2010.

[31] Johansson, Karl Henrik, "The quadruple-tank process: A multivariable laboratory process with an adjustable zero," IEEE Transactions on control systems technology, vol. 8(3), pp. 456-465, 2000. 


\section{BIOGRAPHIES OF AUTHORS}

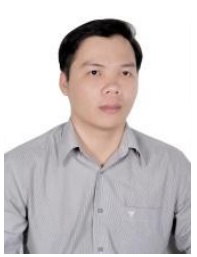

Son Nguyen received his M.Sc. and Ph.D. degrees in the Faculty of Electrical and Electronics Engineering (FEEE) from Ho Chi Minh City University of Technology in 2012 and 2017, respectively. He is currently a Vice-Dean of the Faculty of Electronics Technology, Industrial University of Ho Chi Minh City, Viet Nam. His current research interests include intelligent control, robotics, identification of nonlinear systems, and the internet of things.

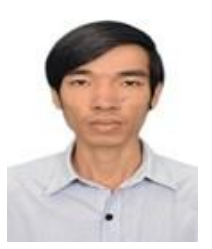

Khanh Nguyen Duy received his M.Sc degree in the Faculty of Telecommunications from Posts and Telecommunications Institute of Technology in 2014. He is currently a Chairman of Student Science Research Club of Faculty of Electronics Technology, Industrial University of Ho Chi Minh City, Viet Nam. His research interests include intelligent systems, machine learning, identification systems, and the internet of thing.

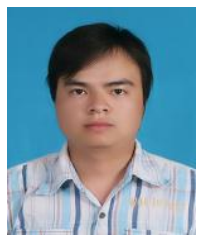

Tran Minh Chinh graduated in 2011 and received Ph.D. degrees in 2016 from Tambov Stade Technical University, Russia. From 9/2016, he works as a teacher in the Faculty of Electronics Technology, Industrial University of Ho Chi Minh City, Viet Nam. His research interests are modern control, intelligent systems, artificially intelligence, machine learning. 